\title{
SLAC T-510: A beam-line experiment for radio emission from particle cascades in the presence of a magnetic field
}

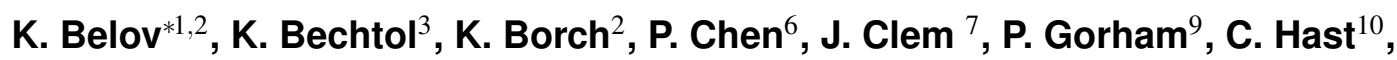 \\ T. Huege ${ }^{11}$, R. Hyneman ${ }^{12}$, K. Jobe ${ }^{10}$, K. Kuwatani ${ }^{2}$, J. Lam ${ }^{2}$, T. Liu ${ }^{6}$, K. Mulrey ${ }^{7}$, \\ J. Nam $^{6}$, C. Naudet ${ }^{1}$, R. Nichol ${ }^{13}$, B.F. Rauch ${ }^{5}$, A. Romero-Wolf ${ }^{1}$, B. Rotter ${ }^{9}$, \\ D. Saltzberg ${ }^{2}$, H. Schoorlemmer ${ }^{9}$, D. Seckel ${ }^{7}$, B. Strutt ${ }^{13}$, A. Vieregg $^{3}$, C. Williams ${ }^{8}$, \\ S. Wissel ${ }^{2}$, A. Zilles ${ }^{14}$
}

${ }^{1}$ Jet Propulsion Laboratory, California Institute of Technology, Pasadena, CA 91109, USA,

E-mail: Konstantin.V.Belov@jpl.nasa.gov.

${ }^{2}$ Dept. of Physics and Astronomy, Univ. of California, Los Angeles, Los Angeles, CA 90095 , USA.

${ }^{3}$ Dept. of Physics, University of Chicago, Chicago, IL, USA.

${ }^{4}$ Dept. of Physics and Astronomy, Univ. of Kansas, Lawrence, KS 66045, USA.

${ }^{5}$ Dept. of Physics, Washington Univ., St. Louis, MO 63130, USA.

${ }^{6}$ Dept. of Physics, Grad. Inst. of Astrophys., \& Leung Center for Cosmology and Particle Astrophysics, National Taiwan University, Taipei, Taiwan.

${ }^{7}$ Dept. of Physics, Univ. of Delaware, Newark, DE 19716, USA.

${ }^{8}$ Dept. of Physics, Stanford University, Stanford, CA, 94305, USA.

${ }^{9}$ Dept. of Physics and Astronomy, Univ. of Hawaii, Manoa, HI 96822, USA.

${ }^{10}$ SLAC National Accelerator Laboratory, Menlo Park, CA, 94025, USA.

${ }^{11}$ Karlsruher Institut für Technologie, Institut für Kernphysik, 76021 Karlsruhe, Germany.

${ }^{12}$ Physics Dept., College of William \& Mary, Williamsburg VA 23187, USA.

${ }^{13}$ Dept. of Physics and Astronomy, University College London, London, United Kingdom.

${ }^{14}$ Karlsruher Institut für Technologie, Institut für Experimentelle Kernphysik, 76128 Karlsruhe, Germany.

We report on the first observations of radio emission from transverse currents induced in a secondary cascade by a magnetic field in dense media. Only the Askaryan effect has been observed in previous experiments in ice, silica sand and in rock salt. These measurements are important because the radio detection of ultra-high energy cosmic rays (UHECRs) relies on models of radio emission from extensive air showers. In order to validate the models we performed an experiment at SLAC National Accelerator Laboratory in 2014 using a high density polyethylene (HDPE) target placed in a magnetic field, yielding results within $36 \%$ of the model predictions.

The 34th International Cosmic Ray Conference,

30 July- 6 August, 2015

The Hague, The Netherlands

\footnotetext{
* Speaker.
} 


\section{Introduction}

Detectors utilizing air fluorescence and ground counting techniques have accumulated significant statistics on UHECRs with energies as high as $3 \times 10^{20} \mathrm{eV}$ [3]. In recent years, radio detection emerged as a viable method that can provide round-the-clock calorimetric measurements of the energy of the UHECRs and the shower development profile, which can be used to obtain chemical composition and inelastic cross-section of the primary particles. The need for an alternative technique is dictated not only by potentially lower deployment and operating costs, but also by a possibility to resolve discrepancies in the data obtained using traditional methods. Balloon-borne and ground-based observatories reported successful detections of the UHECRs using radio [4]. The radio detection technique relies on models of radio emission from extensive air showers. Newly developed formalisms based on first principles $[5,6]$ treat each shower particle as an independent radiator, summing up the emission from all particles in the cascade to obtain the signal received by an observer. This approach does not separate different components of the emission, but for the purpose of simplicity we discuss them separately. Askaryan radiation forms from a charge excess built up in the shower due to pair production, positron absorption and Compton scattering, creating a time-varying current along the shower axis [8]. Such emission has been already measured in accelerator experiments $[9,10,11]$. The Lorentz force also acts on charges in the shower, generating a time-variable transverse current, the Hall current, and subsequent radiation. In case of an extensive air shower induced by an UHECR particle, this radiation will be correlated with the Earth's magnetic field and can be called geomagnetic radiation. The SLAC T-510 experiment is designed to validate the new formalisms based on first principles by conducting measurements of the radio frequency emission from secondary cascades in a controlled laboratory environment.

\section{Experimental setup}

The experiment, SLAC T-510, was performed inside End Station A building at SLAC National Accelerator Laboratory in January-February, 2014. A $1500 \mathrm{~kg}$ HDPE target was constructed from 2 " $\times 4$ " $\times 12$ " rectangular bricks of pure polyethylene with an index of refraction of 1.53 . The target is $396 \mathrm{~cm}$ long by $60 \mathrm{~cm}$ wide by $97 \mathrm{~cm}$ tall at the highest point. Simulations show that the target contains almost all the shower particles. The upper layer bricks are shaped to create a slope of $\sim$ $9.8^{\circ}$ in the forward direction to form a trapezoidal side cross-section. The purpose of the slope is to avoid internal reflection of the emerging radiation at the target boundary. The electron beam enters the target $12 \mathrm{~cm}$ above the floor through a $1.2 \mathrm{~cm}$ thick lead plate. The lead provides pre-radiation that allows us to reduce the overall length of the target. The shower maximum, $X_{\max }$, occurs $\sim 90 \mathrm{~cm}$ into the target. The showers are induced in the target by $4.35 \mathrm{GeV}$ and $4.55 \mathrm{GeV}$ electron bunches with $131 \pm 3 \mathrm{pC}$ mean charge measured at the target, which corresponds to a $\sim 4 \times 10^{18} \mathrm{eV}$ primary cosmic particle energy. The beam current is measured by an integrated charge transformer placed between the beam pipe exit and the target entrance. The systematic uncertainty in the beam charge was obtained by making a series of successive measurements at different places along the beam and does not exceed 3\%. Fig. 1 shows the schematics of the experiment and the target layout. A vertical magnetic field is formed by 15 water cooled coils aligned along the beam axis each carrying up to $\sim 800$ A current of reversible polarity. This allows us to create a mostly vertical 


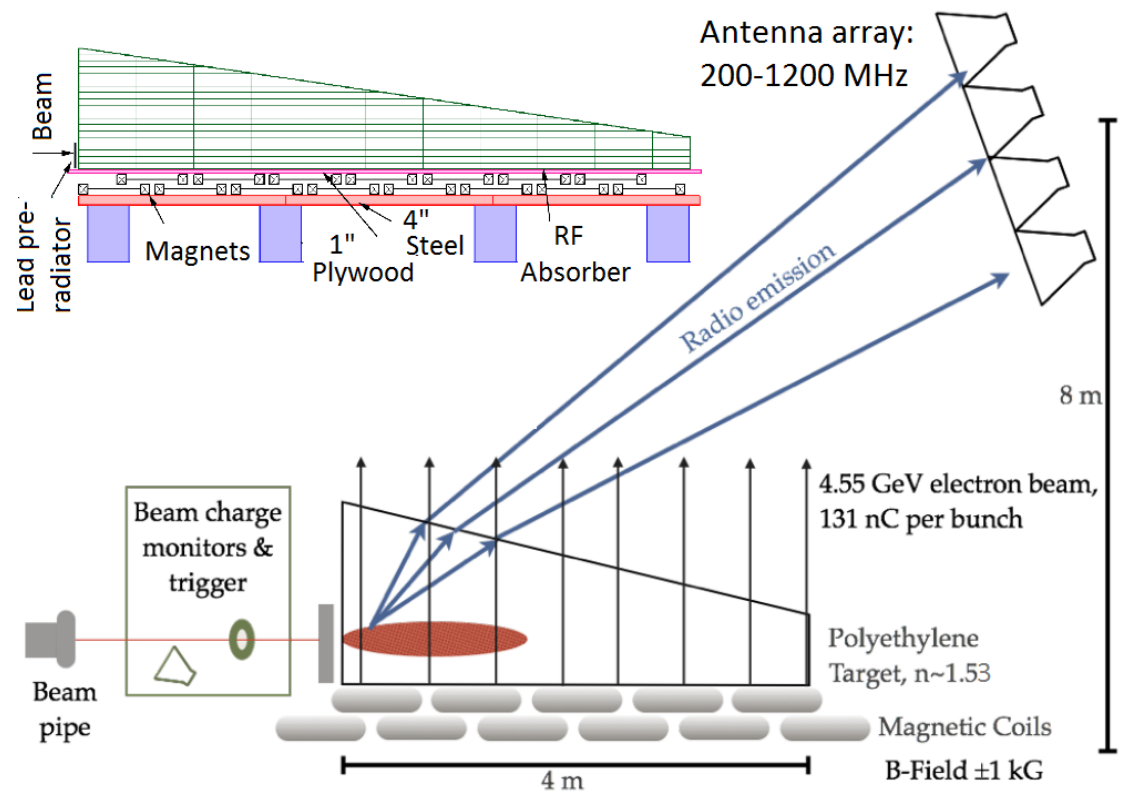

Figure 1: T-510 experiment schematics with target details.

magnetic field of up to $970 \mathrm{G}$ at the beam height around the shower maximum. In order to create a more uniform magnetic field, the coils are assembled in two staggered layers on a 4" thick steel plate. We measured the magnetic field in a $5 \times 5 \mathrm{~cm}$ grid at the beam height across the whole target area. The variation on the magnetic field does not exceed $15 \%$ along the beam line inside the target at the beam height. We estimate the B-field uncertainty to be $5.7 \%$ at every measured location. In order to suppress reflections, the target floor is lined with an RF absorbing blanket and several pieces of ANW-79 absorber are placed at both sides of the target around the shower maximum and at the exit surface of the target. The signal is received by quad-ridged horns borrowed from the ANITA experiment, bicone or log-periodic dipole antennas (LPDAs), see Fig. 2. In three separate

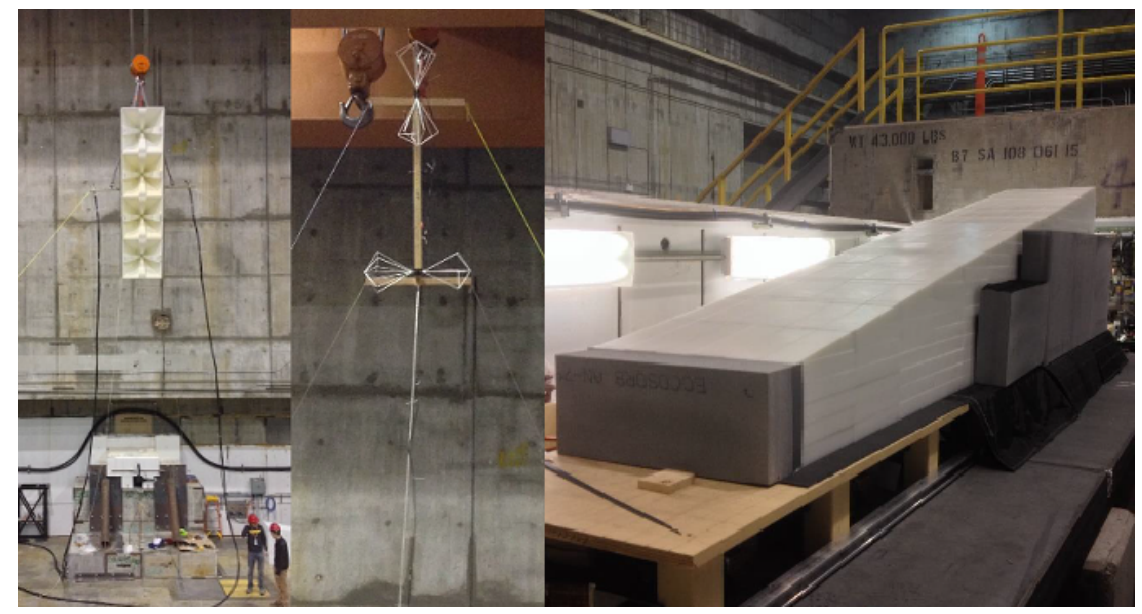

Figure 2: UHF, VHF (bicone) antennas and HDPE target.

measurements four linearly polarized $200-1200 \mathrm{MHz}$ horn antennas sensitive to both polarizations 
were mounted in a vertical line to probe the shape of the wave front, two linearly polarized bicone antennas sensitive to $50-300 \mathrm{MHz}$ were mounted vertically $\sim 2 \mathrm{~m}$ apart and two high frequency LPDA antennas mounted in the same fashion as the bicone antennas cover the frequency range up to $3 \mathrm{GHz}$. The antennas are hung from a crane so they can be moved in all directions The majority of the data taking consists of moving the antennas vertically to sample a slice of the expected Čerenkov cone about $13 \mathrm{~m}$ away from the beginning of the target, and at heights varying from 0 to $12 \mathrm{~m}$ above the beam axis. The DAQ system is triggered by an S-Band horn antenna placed near the exit of the beam pipe to register transition radiation (TR). The TR signal also provides the shotto-shot charge calibration. Waveforms from the UHF horns are recorded on $2.0 \mathrm{GHz}$ oscilloscopes, sampled at $5 \mathrm{GSa} / \mathrm{s}$, while the waveforms from the VHF antennas, LPDAs, and S-band horn are recorded at $10 \mathrm{GSa} / \mathrm{s}$ on $3 \mathrm{GHz}$ oscilloscopes. We only report the measurements done with the UHF horn antennas in this paper. The electric field at the face of the antennas is reconstructed by deconvolving the signal with the frequency-dependent effective height of the quad-ridge horns and the cable loss according to the equation $V=h_{e f f} E$. The change in impedance from free space to a $50 \Omega$ system is accounted for in the effective antenna height. The antenna cross-talk is removed for the horizontally polarized component by averaging the waveforms with opposite polarity in the current flowing through the magnetic coils. The details about the experimental setup and the data processing can be found in [1].

\section{Radio emission models}

The simulations of the T-510 experiment are performed using GEANT4 code [12] altered to include emission from each charged-particle track. The electric field was simulated on a grid normal to the incident electron beam $13 \mathrm{~m}$ from the target entrance. The simulations for the experiment are performed in two stages. The preliminary stage was done before the experiment to determine the optimal experimental set up, including the choice of the target material, required magnetic field strength and the expected signal. The nominal parameters of the beam such as the energy of the primary particles and the beam charge were used at this stage. The radio emission is implemented following Ref. [6]. The second stage of simulations is done after the experiment using the actual beam parameters, measured magnetic field and the actual experimental set up inside the End Station A building. Refraction and demagnification effects at the target surface as well as transition radiation are also taken into account at the final stage. In addition to the previously mentioned formalism, the radio emission is also modeled using Ref. [5] during the final stage. The simulations are performed using a smaller number of electrons per bunch and the results are scaled up to the beam charge. The simulations are described in details in Ref. [2]. The systematic uncertainty between the models does not exceed 3\% in the peak-to-peak amplitudes or in the frequency spectra in the 30-1400 MHz range. Fig. 3 shows the expected magnitude of the electric field, "radio footprint" if a $970 \mathrm{G}$ vertically oriented magnetic field is applied. The pattern is formed due to coherence of the emission from the individual particles of the cascade at the Čerenkov angle. In the presence of a magnetic field the Askaryan effect manifests an asymmetry in the Čerenkov ring. Due to a $\sim 20 \%$ charge imbalance, the whole shower is deflected to one side in the magnetic field, changing the relativistic timing and causing the observed asymmetry. A detailed analysis of the peak-to-peak voltages on different sides of the Čerenkov ring shows that the effect is reproduced 


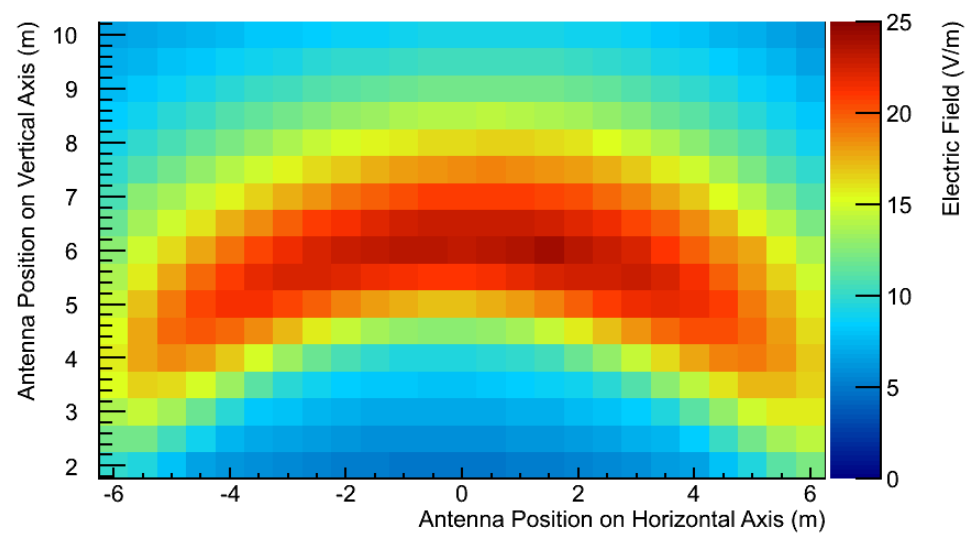

Figure 3: The magnitude of the electric field in a grid $13 \mathrm{~m}$ from the entrance of the beam on target. Simulated at $970 \mathrm{G}$ magnetic field around $X_{\max }, 131 \mathrm{pC}$ charge, $4.35 \mathrm{GeV}$ energy.

very well by our models. The elliptical form of the Čerenkov ring is caused by refraction at the upper flat tapered surface of the target. The ring is cut off on both sides due to the fixed target dimensions. The internal reflections from the target walls as well as the reflections from the structure inside the End Station A building are very difficult to account for and are left out for these simulations. The reflections inside the target ended up being the largest contributor to the systematic uncertainty.

\section{Results}

In the absence of the magnetic field in the target, SLAC T-510 experiment was able to observe the Askaryan signal confirming observations by previous experiments $[9,10,11]$. Askaryan emission is inherently radially polarized, and it appeared as a vertically polarized signal on top of the Čerenkov cone where the measurements were done. The introduction of the vertical magnetic field in the target results in the induction of Hall currents in the cascade. As a result, a horizontal component of the electric field is added to the vertically polarized Askaryan component, effectively rotating the polarization vector. As predicted by the models, the magnitude of the horizontal component depends linearly on the magnetic field strength, see left plot in Fig. 4. It is also not surprising that the Askaryan and "magnetic" signal scales linearly with the beam charge, which is shown in the right plot in Fig. 4. Fig. 5 illustrates that signal polarity flips with the reversal of the magnetic field direction. A scan in the vertical direction across the top of the Čerenkov cone is shown in Fig. 6 and shows very good agreement between the models and the measured "magnetic" signal in three separate frequency bands. The narrowing of the cone at higher frequencies, which is seen in the plots in Fig. 6, confirms the coherent emission model. While the response of the horn antennas goes down to $200 \mathrm{MHz}$, we only use the frequencies above $300 \mathrm{MHz}$ to exclude the region where the antenna calibration is more uncertain. 

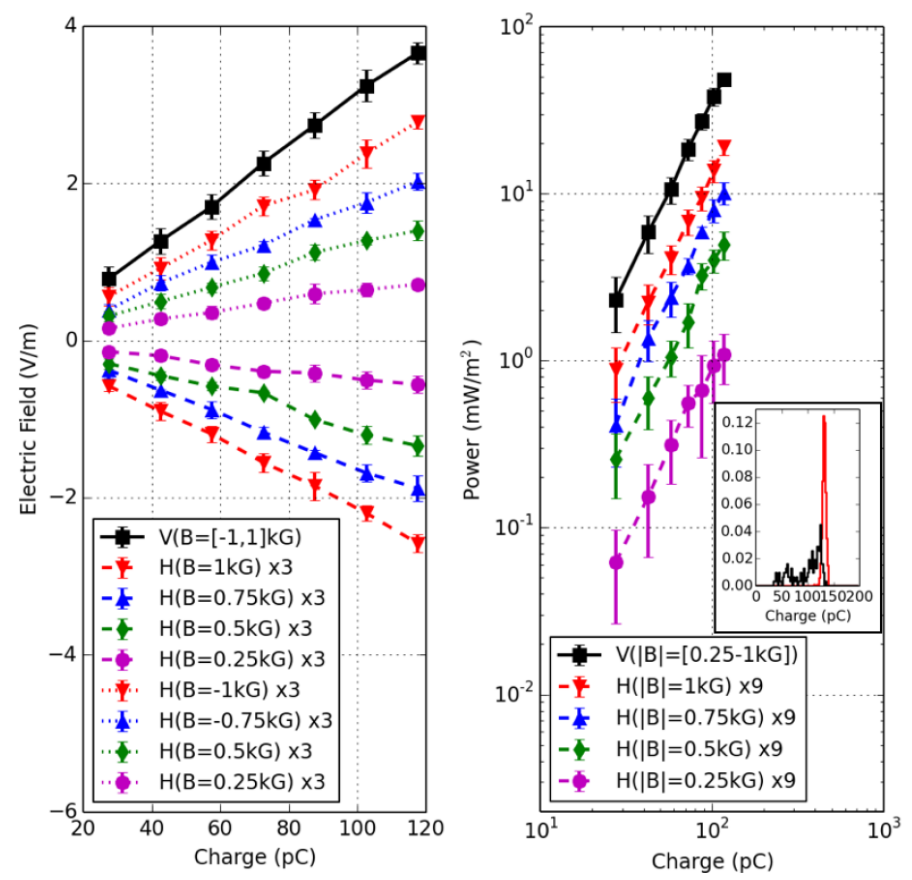

Figure 4: The electric field peak amplitude (left) and intensity (right) at the top of the Čerenkov cone for the vertically polarized (black, squares solid) and horizontally polarized channels (scaled by 3 left; by 9 right) for different magnetic fields.

\section{Discussion}

SLAC T-510 confirmed the Askaryan radiation for particle cascades in dense media that was reported by previous experiments $[9,10,11]$ and also confirmed that the emission scales linearly with the beam charge. We observed the expected emission component due to transverse electric currents induced in the target by cascade particle deflection in an applied magnetic field and found that this magnetic component scales linearly with field strength in agreement with models. As predicted by the models, the polarity of the emission signal reverses with the change of the magnetic field polarity, confirming the time-varying Hall current nature of the magnetic emission component. The magnetic current induction manifests itself as the polarization vector rotation of the total emission from the shower. The absolute amplitude of the measured electric field agrees with models to within $36 \%$ with the largest systematic uncertainty coming from reflections in the target. The reflections show up as a delayed signal, altering the pulse shape and frequency content. Because filtering the reflections out by windowing the signal would introduce a bias in the power spectrum, we decided to include the reflections in the systematic error instead and leave the improvements for future work. The frequency dependence of the Čerenkov cone shape is also measured and is in very good agreement with the models. A slight asymmetry of the observed Čerenkov cone may be due to diffraction effects in the target that are seen at different angles at the top and at the bottom within the measurement range as well as due to the reflections, as they exhibit a delay rate. These effects are also difficult to model and they are left out of this discussion. By validating the models of radio emission from extensive air showers, the SLAC T-510 experiment provides a framework 


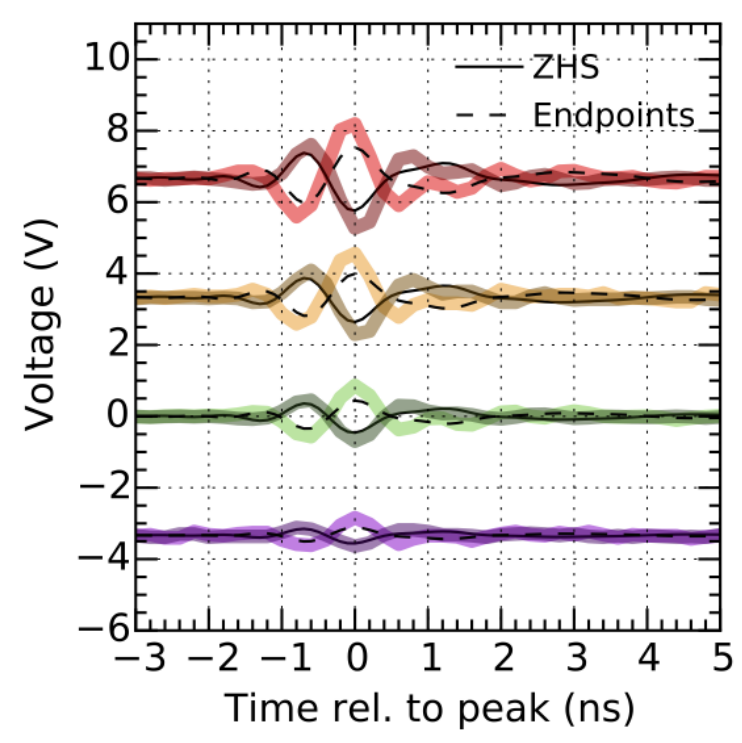

Figure 5: Impulse dependence on magnetic field and polarity. Simulated: magenta $-250 \mathrm{G}$, green $-500 \mathrm{G}$, yellow $-750 \mathrm{G}$, red $-970 \mathrm{G}$. Data: black. DC offset added to visually separate the impulses.

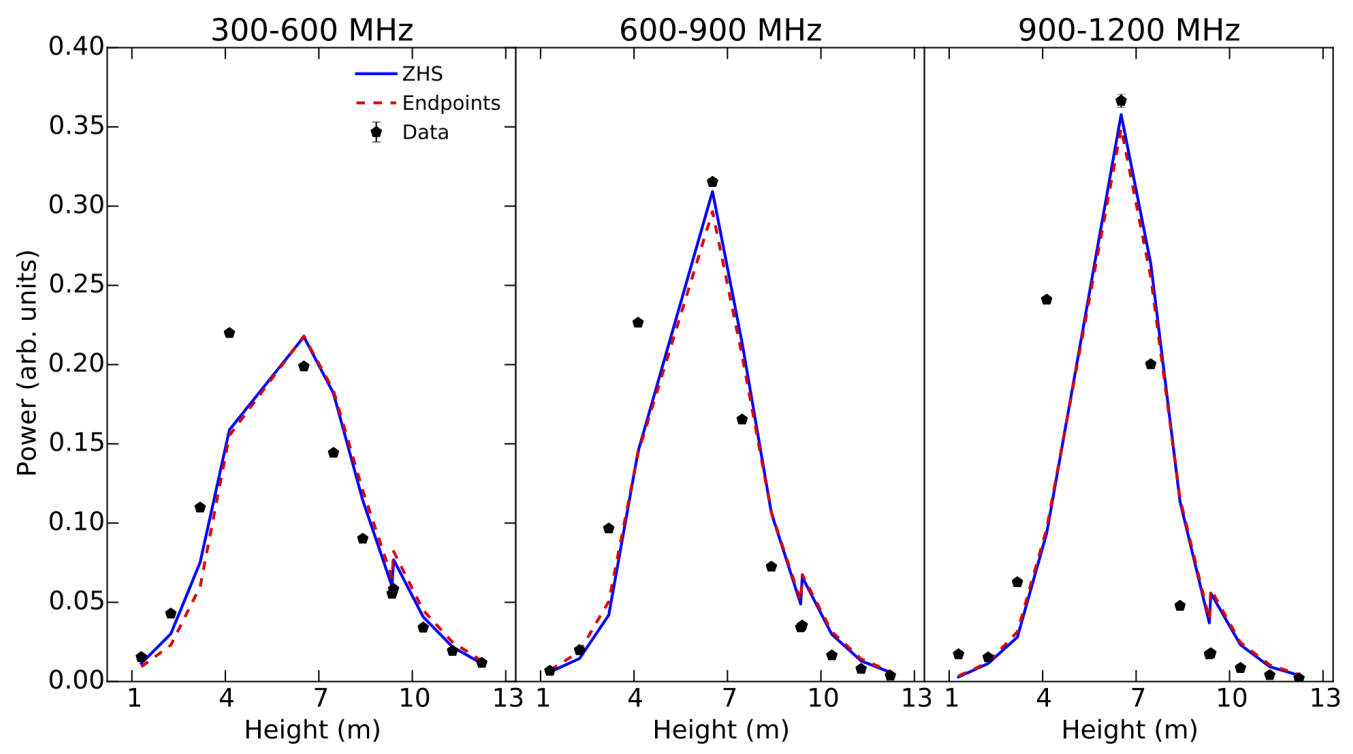

Figure 6: Radio emission beam patterns for magnetic emission as measured in a vertical slice aligned with the beam in three frequency ranges.

for measuring the energy UHECRs using radio technique.

\section{Acknowledgments}

The authors thank SLAC National Accelerator Center for providing facilities and support and especially Janice Nelson and Carl Hudspeth for their support and dedication that made T-510 possible. Work supported in part by Department of Energy contract DE-AC02-76SF00515, the Taiwan 
Ministry of Science and Technology under project number MOST103-2119-M-002-002 and others. Part of the research was carried out at the Jet Propulsion Laboratory, California Institute of Technology, under a contract with the National Aeronautics and Space Administration.

\section{References}

[1] S.Wissel et al., Measurements, system response, and calibration of the SLAC T-510 Experiment, see contribution 826 of this conference.

[2] A. Zilles et al., Modelling of radio emission in the SLAC T-510 Experiment using microscopic Geant4 simulations, see contribution 660 of this conference.

[3] D. J. Bird, S. C. Corbato, H. Y. Dai, J. W. Elbert, K. D. Green, M. A. Huang, D. B. Kieda, S. Ko, C. G. Larsen, E. C. Loh, M. Z. Luo, M. H. Salamon, J. D. Smith, P. Sokolsky, P. Sommers, J. K. K. Tang, and S. B. Thomas, Detection of a cosmic ray with measured energy well beyond the expected spectral cutoff due to cosmic microwave radiation, ApJ 441 (Mar., 1995) 144-150.

[4] T. Huege, The renaissance of radio detection of cosmic rays, Braz J Phys 44 (2014), no. 5 520-529.

[5] C. W. James, H. Falcke, T. Huege, and M. Ludwig, General description of electromagnetic radiation processes based on instantaneous charge acceleration in "endpoints", Phys. Rev. E 84 (Nov, 2011).

[6] Alvarez-Muñiz, Jaime and Romero-Wolf, Andrés and Zas, Enrique, Čerenkov radio pulses from electromagnetic showers in the time domain, Phys. Rev. D 81 Jun, 2010

[7] J. Alvarez-Muñiz, W. R. Carvalho Jr., and E. Zas, Monte carlo simulations of radio pulses in atmospheric showers using ZHAireS, Astropart. Phys. 35 (2012), no. 6325 - 341.

[8] G. A. Askaryan, Excess Negative Charge of an Electron-Photon Shower And Its Coherent Radio Emission, Soviet Physics JETP 14 (1962) 441.

[9] D. Saltzberg, P. Gorham, D. Walz, C. Field, R. Iverson, A. Odian, G. Resch, P. Schoessow, and D. Williams, Observation of the askaryan effect: Coherent microwave Čerenkov emission from charge asymmetry in high-energy particle cascades, Phys. Rev. Lett. 86 (Mar, 2001) 2802-2805.

[10] P. W. Gorham, S. W. Barwick, J. J. Beatty, D. Z. Besson, W. R. Binns, C. Chen, et al., Observations of the askaryan effect in ice, Phys. Rev. Lett. 99 (Oct, 2007) 171101.

[11] P. W. Gorham, D. Saltzberg, R. C. Field, E. Guillian, R. Milinčić, P. Miočinović, D. Walz, and D. Williams, Accelerator measurements of the askaryan effect in rock salt: A roadmap toward teraton underground neutrino detectors, Phys. Rev. D 72 (Jul, 2005) 023002.

[12] S. Agostinelli, J. Allison, K. Amako, J. Apostolakis, H. Araujo, P. Arce, et al., Geant4 simulation toolkit, Nucl. Instrum. Meth. A 506 (2003), no. 3250 - 303.

[13] E. Zas, F. Halzen, and T. Stanev, Electromagnetic pulses from high-energy showers: Implications for neutrino detection, Phys. Rev. D 45 (Jan, 1992) 362-376.

[14] T. Huege, M. Ludwig, and C. W. James, Simulating radio emission from air showers with coreas, AIP Conf. Proc. 1535 (2013), no. 1 128-132. 